\title{
Comparative Degradation Kinetic Studies of Tetrabutyl- ammonium Tribromide (TBATB) and Cetyltrimethyl- ammonium Tribromide(CTMATB) - A Route to Reactivity Assessment for Solvent-Free Reactions
}

\author{
NEIVOTSONUO B. KUOTSU ${ }^{* 1}$, CHUBAAKUM PONGEN ${ }^{2}$, \\ TOVISHE PHUCHO ${ }^{2}$ and UPASANA B. SINHA ${ }^{2}$
}

${ }^{1}$ Department of Chemistry, Kohima Science College, Jotsoma-797002, Kohima, Nagaland, India

${ }^{2}$ Department of Chemistry, Nagaland University, Lumami-798627, Nagaland, India

solo_berna@yahoo.com

Received 17 October 2014 / Accepted 8 November 2014

\begin{abstract}
Tetrabutylammonium tribromide (TBATB) and cetyltrimethylammonium tribromide (CTMATB) are important alternatives to the hazardous reagent bromine $\left(\mathrm{Br}_{2}\right)$ because of their efficiency in various chemical transformations, especially organic bromination reactions. In the context of green chemistry, development of solvent-free reaction protocols is much needed because of the hazards posed by organic solvents. In order to assess the comparative behavior of these tribromides in solvent-free reactions at elevated temperatures, thermogravimetric studies came in as a useful tool. Thus, the kinetics of the thermal degradation of TBATB and CTMATB were investigated through thermogravimetric analysis and the thermal stability order of the two bromides based on TG followed the order: CTMATB>TBATB. Non isothermal multiple heating rate model free methods Ozawa-Flynn-Wall and Kissinger methods are used to calculate degradation activation energy of the tribromides. The reaction mechanism based on activation energy calculation at different conversions has been proposed
\end{abstract}

Keywords: Tetrabutylammonium tribromide, Cetyltrimethylammonium tribromide, Thermal analysis, Brominations, Solvent-free, Environmentally benign

\section{Introduction}

Acknowledging the importance of bromo-organic compounds ${ }^{1-2}$ and the need for environmentally benign brominating reagents, substantial developments have been made through the synthesis and use of quaternary ammonium tribromides (QATBs) as alternatives of bromine $e^{3-10}$. Among the numerous tribromides that have been synthesized so far, tetrabutylammonium tribromide (TBATB) and cetyltrimethylammonium tribromide (CTMATB) have been found to be useful for a number of different types of reactions, including solvent-free brominations conducted at elevated temperature and under microwave 
conditions $^{3,5}$. Ability for any reagent to perform at high temperature greatly broadens the scope of the reagent for other solvent-free reactions and thereby it becomes important to understand their degradation pattern at elevated temperature. It has been observed that in the case of QATBs, other than a brief study on CTMATB ${ }^{11}$ there seems to be no report on their thermal analysis, especially in terms of a comparative assessment. Acknowledging the importance of such kind of a study, cetyltrimethylammonium tribromide (CTMATB) and tetrabutylammonium tribromide (TBATB) have been taken as candidates for estimating the kinetic parameters of degradation processes such as activation energies (Ea) and the Arrhenius pre-exponential factor $(\mathrm{A})^{15-23}$. Accordingly, this paper deals with the comparative thermal and kinetic study of TBATB and CTMATB using simultaneous thermal analysis by the Kissinger ${ }^{12}$ and Flyn-Wall-Ozawa methods ${ }^{13-14}$. The Kissinger method derives from the Arrhenius Equation, which correlates the rate constant with the activation energy, through an equation:

$$
\mathrm{K}=\mathrm{A} \operatorname{Exp}[-\mathrm{Ea} / \mathrm{RT}]
$$

Where, $k$ is the rate constant; $A$ is the pre-exponential factor also known as frequency factor; $E_{a}$ is the activation energy; $R$ is gas constant and $T$ is the temperature. The Kissinger method can be used in calculating activation energy at different heating rate $(\beta)$ which is given by the equation (2) as:

$$
\ln \left(\beta / \mathrm{T}^{2}{ }_{\max }\right)=\ln \mathrm{AR} / \mathrm{Ea}+\ln \left(\mathrm{n}\left[1-\alpha_{\max }\right) \mathrm{n}-1\right)-\left(\mathrm{E} / \mathrm{RT}_{\max }\right)
$$

Where $\beta$ is heating rate, $T_{\max }$ is temperature related to maximum reaction rate, $A$ is the preexponential factor, $\alpha_{\max }$ is the maximum degradation fraction, $n$ is reaction order. Plotting $\ln \left(\beta / \mathrm{T}^{2}{ }_{\max }\right)$ versus ( $\left.1000 / \mathrm{T}_{\max }\right)$ gives activation energy from slope.

The Kissinger plot thus says that for a given DSC curve with the heating rate, $\beta$, one observes the maximum reaction rate at the peak temperature, $T_{\mathrm{p}}$; for a set of DSC curves with different heating rates, one can plot the quantity of $\ln \left(\beta / T_{\mathrm{p}}{ }^{2}\right)$ against $1 / T_{\mathrm{p}}$ to obtain the Kissinger plot. From the slope of the Kissinger plot, one in turn obtains the activation energy, $E_{\mathrm{a}}$; further from the intercept one obtains the pre-exponential factor, $A$, as well.

The Flynn-Wall-Ozawa method is an integral method that can be used to determine the activation energy without the knowledge of reaction mechanism, pre-exponential factor (A) and activation energy $\left(E_{a}\right)$ do not depend on degradation fraction, but they depend on the temperature. This method uses equation (3) as:

$$
\log g(\alpha)=\log (\mathrm{AEa} / \mathrm{R})-\log \beta+\log \mathrm{P}(\mathrm{E} / \mathrm{RT})
$$

Doyle approximation is used and equation (3) can be obtained.

Where $\beta$ is the heating rate and $g(\alpha)$ is the conversion temperature. The plot of $\log \beta$ versus $1000 / T$ should be linear with the slope $E_{a} / R$ from which $E_{a}$ can be calculated.

$$
\log \beta=\log (\mathrm{AEa} / \mathrm{R})-\log g(\alpha)-2.314-0.4567(\mathrm{E} / \mathrm{RT})
$$

\section{Experimental}

All reagents of highest purity were purchased from commercial sources and used without further purification. Melting points were determined in open capillaries and are uncorrected.

\section{Synthesis of tetrabutylammonium tribromide(TBATB)}

$0.057 \mathrm{~g}(0.53 \mathrm{mmol})$ sodium carbonate $\mathrm{Na}_{2} \mathrm{CO}_{3}$ was added to $50 \% \mathrm{H}_{2} \mathrm{O}_{2},(10 \mathrm{~mL} ; 47 \mathrm{mmol})$ and the mixture was stirred at room temperature for about 5 minutes until $\mathrm{Na}_{2} \mathrm{CO}_{3}$ completely dissolved and the solution attained a clear colourless solution . To this a solution of $5 \mathrm{~g} \mathrm{(15.53}$ $\mathrm{mmol})$ tetrabutyl ammonium bromide (TBAB) and $3.7 \mathrm{~g}(31.90 \mathrm{mmol})$ potassium bromide 
$(\mathrm{KBr})$, both dissolved in $50 \mathrm{~mL}$ of water was added. To the resultant reaction mixture, 50 $\mathrm{mL}$ of $2 \mathrm{M} \mathrm{H}_{2} \mathrm{SO}_{4}$ was added in small portions, upon which the precipitate appeared immediately. The mixture was allowed to be stirred for further $1 \mathrm{~h}$ whereby a bright yellow coloured compound precipitated out completely. The compound was filtered under suction using Whatman-40 filter paper and dried in vacuum desiccators using self-indicating coarse silica gel. It was further recrystallized in acetonitrile, isolated by suction filtration and dried in vacuo yielding $7.38 \mathrm{~g}$ (98\%) of the pure compound.

\section{Synthesis of cetyltrimethylammonium tribromide(CTMATB)}

$0.057 \mathrm{~g}$ ( $0.53 \mathrm{mmol}$ ) sodium carbonate $\mathrm{Na}_{2} \mathrm{CO}_{3}$ was added to $50 \% \mathrm{H}_{2} \mathrm{O}_{2}(10 \mathrm{~mL} ; 47$ mmol ) and the mixture was stirred at room temperature for about 5 minutes until $\mathrm{Na}_{2} \mathrm{CO}_{3}$ completely dissolved and the solution attained a clear colourless solution. To this a solution of $5 \mathrm{~g}$ (15.53 mmol) cetyltrimethylammonium bromide (CTMAB) and $3.7 \mathrm{~g}$ (31.90 mmol) potassium bromide $(\mathrm{KBr})$, both dissolved in $50 \mathrm{ml}$ of water was added. To the resultant reaction mixture, $50 \mathrm{~mL}$ of $2 \mathrm{M} \mathrm{H}_{2} \mathrm{SO}_{4}$ was added in small portions, upon which the precipitate appeared immediately. The mixture was allowed to be stirred for further $1 \mathrm{~h}$ whereby a bright yellow coloured compound precipitated out completely. The compound was filtered under suction using Whatman-40 filter paper and dried in vacuum desiccators using self-indicating coarse silica gel. It was further recrystallized in acetonitrile, isolated by suction filtration and dried in vacuo. The yield of the isolated compound was $7.12 \mathrm{~g}$ (99\%).

\section{Thermogravimetric analysis}

Thermogravimetric (TG) experiments were conducted on a Perkin Elmer STA-6000 (Simultaneous Thermal Analyser) 521A7091405 instrument. Samples weighing about $12 \mathrm{mg}$ were taken in the crucible and heated from 30 to $300{ }^{\circ} \mathrm{C}$ at a heating rate of $5,10,15$ and $20{ }^{\circ} \mathrm{C} / \mathrm{min}$ in nitrogen atmosphere.

\section{Data processing and activation energy calculation}

TG curves were analyzed by using Pyris software from TG Analyzer and data was used in MS Excel software to calculate activation energy (Ea) by the method of least square. Activation energy of samples was calculated by "model free" iso-conversional methods. Kissinger method was used in calculating activation energy at different heating rate $(\beta)$ which is given by the equation (2):

$$
\ln \left(\beta / \mathrm{T}_{\text {max }}^{2}\right)=\ln A R / E a+\ln \left(\mathrm{n}\left[1-\alpha_{\max }\right) \mathrm{n}-1\right)-\left(\mathrm{Ea} / \mathrm{RT}_{\max }\right)
$$

Where $\beta$ is heating rate, $T_{\max }$ is temperature related to maximum reaction rate, $A$ is preexponential factor, $\alpha_{\max }$ is maximum degradation fraction, $\mathrm{n}$ is reaction order. Plotting $\ln \left(\beta / \mathrm{T}^{2}{ }_{\max }\right)$ versus $\left(1000 / \mathrm{T}_{\max }\right)$ gives activation energy from slope. Flyn-Wall-Ozawa method was also used to determined activation energy at a fixed conversion with the slope of such a line being $-0.4567 \mathrm{Ea} / \mathrm{RT}$

\section{Results and Discussion}

\section{Comparative TG and DTG studies}

TG curves for TBATB and CTMATB at different heating rates in nitrogen atmosphere are shown in Figures 1-2. The shapes of TG curves are quite similar, shifting towards higher temperatures at higher heating rates. This is probably due to the heat transfer lag with increased heating rate. The initial decomposition temperature of TBATB and CTMATB occurs around $174{ }^{\circ} \mathrm{C}$ and $176{ }^{\circ} \mathrm{C}$ respectively. Although the values differ slightly the thermal stability of the samples follows the order: CTMATB $>$ TBATB as indicated by Figure 3. 
The decomposition of TBATB and CTMATB occur in single step as confirmed by DTG curves (Figure 4). The main decomposition of TBATB occurs around $230{ }^{\circ} \mathrm{C}$ with a percentage mass of $3 \%$ remaining as unburned whereas in the case of CTMATB it occurs around $231{ }^{\circ} \mathrm{C}$. The higher decomposition value again reiterates the higher stability of cetyltrimethylammonium tribromide.
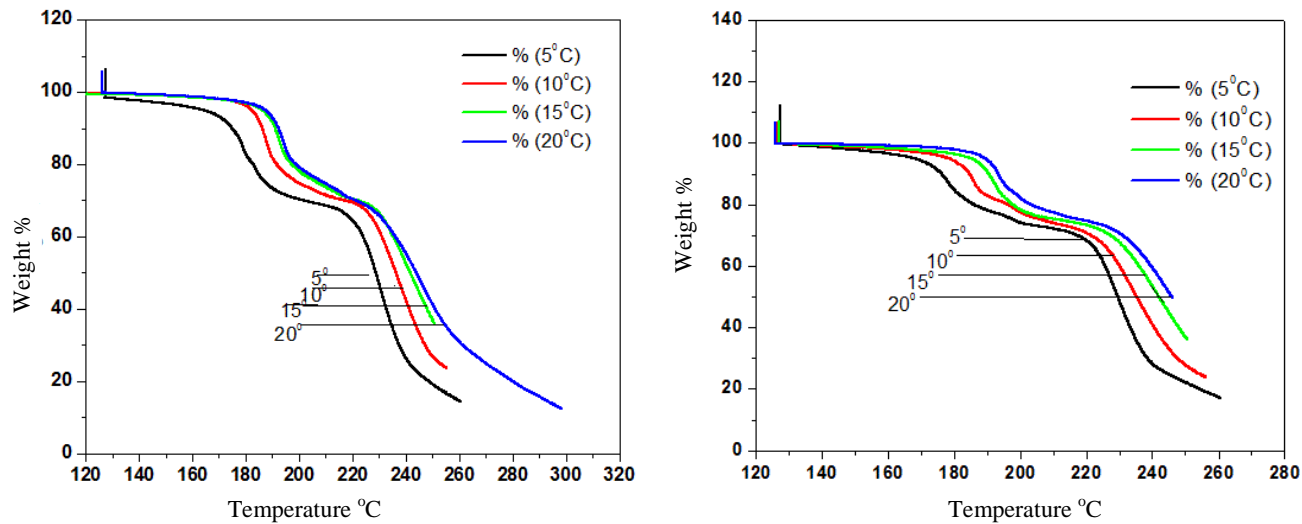

Figure 1. TG curve of TBATB at 5,10,15 and $20{ }^{\circ} \mathrm{C}$

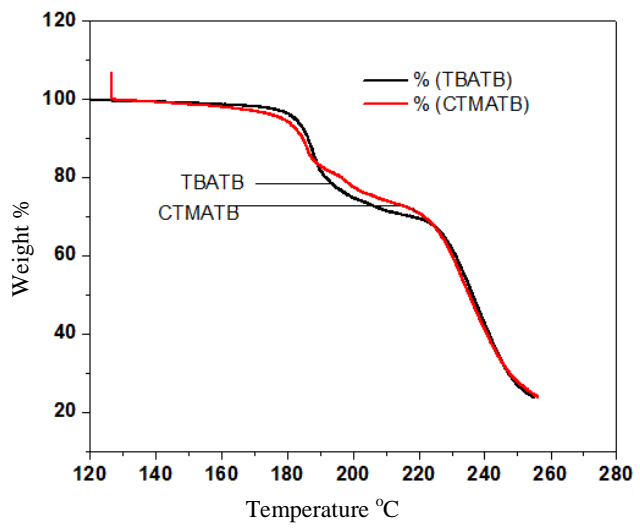

Figure 2. TG curve of CTMATB at 5,10,15 and $20{ }^{\circ} \mathrm{C}$

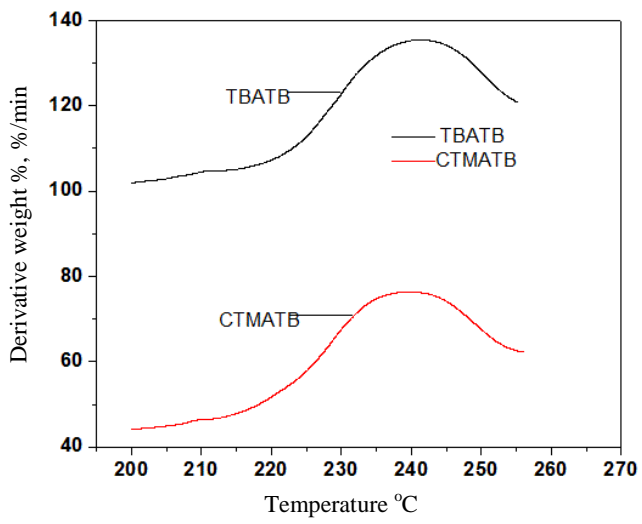

Figure 3. TG curve of TBATB and CIMATB at $10{ }^{\circ} \mathrm{C} / \mathrm{min}$

Figure 4. DTG curves of TBATB and CIMATB at $10{ }^{\circ} \mathrm{C} / \mathrm{min}$

\section{Comparison of degradation activation energies of the two bromides}

Activation energies for TBATB and CTMATB were calculated using Kissing method at varying heating rates $(\beta)$ and are given in Table 1 and 2. The linear plots of Kissinger method for TBATB and CTMATB are given in Figure 5. Activation energies for TBATB and CTMATB were calculated using $\mathrm{O}-\mathrm{F}-\mathrm{W}$ procedure at varying degree of conversions and are given in Table 3. The iso-conversional plot of O-F-W show a general trend of activation energy. For illustration iso-conversional plot of O-F-W for TBATB and CTMATB are shown in Figure 6 and 7. From the figure we observed that the fitted lines for the two bromides are almost parallel which indicates the activation energy at different conversions and consequently, implying the possibility of single reaction mechanism ${ }^{23}$. 
Table 1. Calculation of activation energies for decomposition of TBATB at varying degree of conversions

\begin{tabular}{cccccccc}
\hline$\beta$ & $\ln \beta$ & $\mathrm{T}_{\max }$ & $\mathrm{T}_{\max } \mathrm{K}$ & $\mathrm{T}_{\max } \mathrm{sq}$ & $\beta / \mathrm{T}_{\max } \mathrm{sq}$ & $\ln \beta / \mathrm{T}_{\max } \mathrm{sq}$ & $1000 / \mathrm{T}_{\max }$ \\
\hline 278 & 5.627621 & 228 & 501 & 251001 & 0.001108 & -6.80559109 & 1.99600798 \\
283 & 5.645447 & 229 & 502 & 252004 & 0.001123 & -6.79175334 & 1.99203187 \\
288 & 5.66296 & 230 & 503 & 253009 & 0.001138 & -6.77821986 & 1.98807157 \\
293 & 5.680173 & 230 & 503 & 253009 & 0.001158 & -6.76100773 & 1.98807157 \\
\hline
\end{tabular}

Table 2. Calculation of activation energies for decomposition of CTMATB at varying degree of conversions

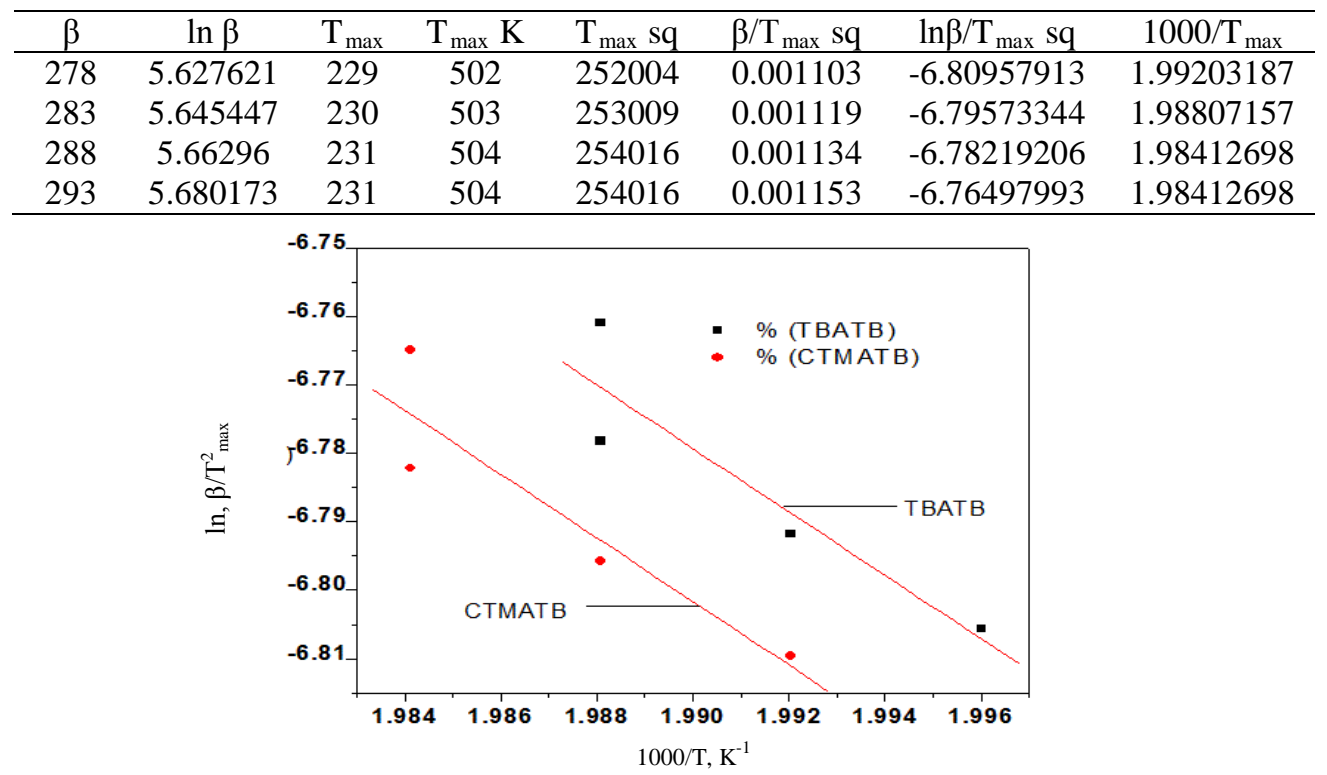

Figure 5. Plot of Kissinger method for TBATB and CTMATB at varying degree of conversions

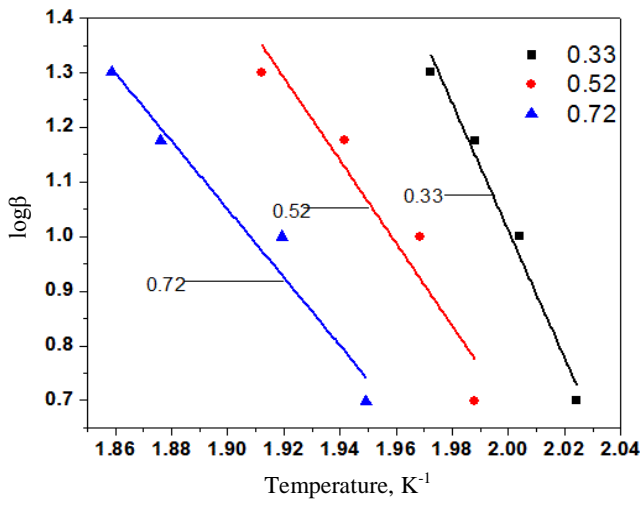

Figure 6. Iso-conversional plot of O-F-W method for TBATB at varying degree of conversions

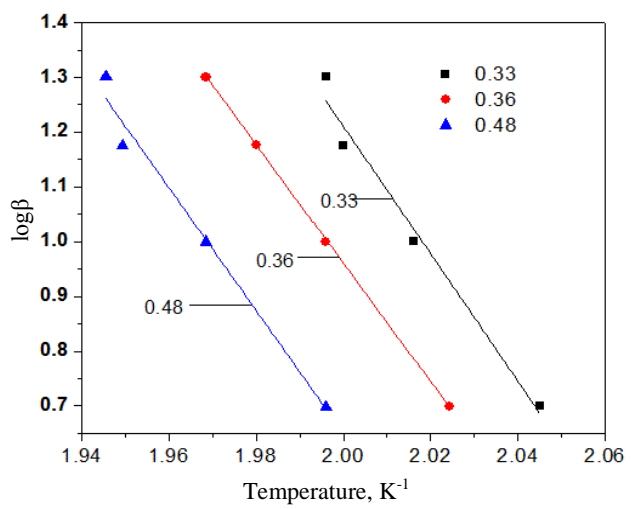

Figure 7. Iso-conversional plot of O-F-W method for CTMATB at varying degree of conversions 
It can be seen from the Table 3 the activation energies calculated by iso-conversional method shows that the degree of conversion i.e. at 0.33 the Ea values for CTMATB was greater than that of TBATB. This means that the initial thermal stability of CTMATB was comparatively higher than TBATB which also matches well with the initial thermal degradation order observed for TG curves. The values calculated by Kissinger method for TBATB and CTMATB are $0.0936 \mathrm{kJmol}^{-1}$ and $0.0947 \mathrm{kJmol}^{-1}$ respectively. The order of E values calculated by Kissinger method was same as evaluated by initial thermal degradation order i.e. CTMATB>TBATB.

Table 3. Activation energies for TBATB and CTMATB calculated by O-F-W method at varying degree of conversions

\begin{tabular}{cccc}
\hline$\alpha$ & TBATB & $\alpha$ & CTMATB \\
\hline 0.33 & 2.35 & 0.33 & 2.36 \\
0.52 & 0.997 & 0.36 & 2.03 \\
0.72 & 0.667 & 0.48 & 2.15 \\
\hline
\end{tabular}

\section{Acknowledgement}

The author (NK) is grateful to Bendang STA, Nagaland University, Lumami, for recording the TGA data.

\section{Conclusion}

TBATB and CTMATB have been synthesized by environmental friendly method. The thermal stability of the synthesized reagent has then been studied in order to understand the basic theory and kinetics of thermal degradation of TBATB and CTMATB.

- On the basis of initial thermal decomposition temperature calculated from TG curves, the thermal stability of samples follows the order CTMATB>TBATB.

- The activation energy values calculated for the tribromides also shows that CTMATB is more stable than TBATB.

- The thermal and kinetic data presented in this study would help to find out new applications of these tribromides either as binary or ternary blends.

\section{References}

1. Anastas P T and Warner J C, Green Chemistry: Theory and Practice, Oxford Univ. Press, 2000.

2. Thematic Issue on Green Chemistry, Chemical Reviews, 2007, 107, 2167-2708.

3. Chaudhuri M K, Bora U, Dehury S K, Dey D, Dhar S S, Kharmawphlang W, Choudary B M and Lakshmi Kantan M, International Publication Number WO 2004/054962 A1, International Publication Date 01. 07. 2004.

4. Bora U, Chaudhuri M K, Dey D and Dhar S S, Pure Appl Chem., 2001, 73(1), 93-102.

5. Kar G, Saikia A, Bora U, Dehury S K, Chaudhuri M K, Tetrahedron Lett., 2003, 44(24), 4503-4505; DOI:10.1016/S0040-4039(03)01015-3

6. Jamir L, Alimenla B, Kumar A, Sinha D and Sinha U B, Synth Commun., 2011, 41(1), 147-155; DOI:10.1080/00397910903531912

7. Kavala V, Naik S and Patel B K, J Org Chem., 2005, 70(11), 4267-4271; DOI:10.1021/jo050059u

8. $\quad$ Li Z Sun X, Wang L, Li Y, Ma Y, J Braz Chem Soc., 2010, 21(3), 496-501; DOI:10.1590/S0103-50532010000300015 
9. Kumar A, Alimenla B, Jamir L, Sinha D and Sinha U B, Org Commun., 2011, 4, 1-8.

10. Alimenla B, Kuotsu B, Sinha U B, Chem Sci Trans., 2014, 3(2), 826-832; DOI:10.7598/cst2014.795

11. Bose G, Li Y, Bhujarbarua M, Kalita D and Khan A T, Chem Lett., 2001, 30(4), 290291; DOI:10.1246/cl.2001.290

12. Kissinger H E, Anal Chem., 1957, 29(11), 1702-1706; DOI:10.1021/ac60131a045

13. Wang D, Das A, Leuteritz A, Bolt R, Haubler L, Wagenknecht U and Heinrich G, Polym Degrad Stab., 2011, 96(3), 285-290;

DOI:10.1016/j.polymdegradstab.2010.03.003

14. Fraga F and Niinez E R, J Appl Polym Sci., 2001, 80(5), 776-782; DOI:10.1002/1097-4628(20010502)80:5<776::AID-APP1154>3.0.CO;2-8

15. Badia J D, Santonja Blasco L, Morina R and Greus R, Polym Degrad Stab., 2010, 95(11), 2192-2199; DOI:10.1016/j.polymdegradstab.2010.06.002

16. Hamciuc C, Vlad-Bubulac T, Petreus O and Lisa G, Eur Polym J., 2007, 43(3), 980988; DOI:10.1016/j.eurpolymj.2006.12.018

17. Liu B Y, Zhao X H, Wang X H, Wang F S, J Appl Polym Sci., 2003, 90(4), 947-953; DOI:10.1002/app.12659

18. Sun J T, Huang Y D, Gong G F, Cao H L, Polym Degrad Stab., 2006, 91(2), 339346; DOI:10.1016/j.polymdegradstab.2005.04.037

19. Meng X, Huang Y D, Yu H and Lv Z, Polym Degrad Stab., 2007, 92(6), 962-967; DOI:10.1016/j.polymdegradstab.2005.04.037

20. Switala-Zeliazkow M, Polym. Degrad. Stab., 2006, 91(6), 1233-1239; DOI:10.1016/j.polymdegradstab.2005.09.006

21. Burnham A K, Thermochim. Acta, 2000, 355(1-2), 165-170; DOI:10.1016/S00406031(00)00446-9

22. Vyazovkin S, Thermochim Acta, 2000, 355(1-2), 155-163; DOI:10.1016/S00406031(00)00445-7

23. Arora S, Lal S, Kumar S, Kumar M and Kumar M, Arch Appl Sci Res., 2011, 3(3), 188-201. 